\title{
Kantorovich-type operators associated with a variant of Jain operators
}

\author{
Octavian Agratini and Ogun Doğru
}

Dedicated to Professor Gheorghe Coman on the occasion of his 85th birthday, with high esteem.

\begin{abstract}
This note focuses on a sequence of linear positive operators of integral type in the sense of Kantorovich. The construction is based on a class of discrete operators representing a new variant of Jain operators. By our statements, we prove that the integral family turns out to be useful in approximating continuous signals defined on unbounded intervals. The main tools in obtaining these results are moduli of smoothness of first and second order, K-functional and BohmanKorovkin criterion.
\end{abstract}

Mathematics Subject Classification (2010): 41A36, 41A25.

Keywords: Linear positive operator, Jain operator, modulus of smoothness, K-functional, Lipschitz function.

\section{Introduction}

The starting point of the paper is a class of operators introduced by G.C. Jain [8]. The construction is based on a Poisson-type distribution with two parameters given by

$$
w_{\beta}(k ; \alpha)=\frac{\alpha}{k !}(\alpha+k \beta)^{k-1} e^{-(\alpha+k \beta)}, k \in \mathbb{N}_{0},
$$

for $\alpha>0$ and $|\beta|<1$. With the help of Lagrange inversion, in [1, Lemma 1] was proved

$$
\sum_{k=0}^{\infty} w_{\beta}(k ; \alpha)=1 .
$$

Considering $w_{\beta}(k ; 0)=\delta_{k, 0}$, Kronecker's delta symbol, Jain defined the operators

$$
\left(P_{n}^{[\beta]} f\right)(x)=\sum_{k=0}^{\infty} w_{\beta}(k ; n x) f\left(\frac{k}{n}\right), x \geq 0,
$$


where $\beta \in[0,1)$ and $f \in C\left(\mathbb{R}_{+}\right)$whenever the above series is convergent. Here $C\left(\mathbb{R}_{+}\right)$ stands for the space of real-valued continuous functions defined on $\mathbb{R}_{+}=[0, \infty)$. Clearly, $P_{n}^{[\beta]}, n \in \mathbb{N}$, are linear and positive operators.

In recent years, the investigation of these operators have been invigorated obtaining new properties as well as various generalizations. For a brief synthesis, [2] can be consulted.

Set $\mathbb{N}_{0}=\{0\} \cup \mathbb{N}$. We consider the functions $e_{0}(x)=1, e_{m}(x)=x^{m}, x \geq 0$. The first three monomials represent the so-called Korovkin test functions, having an essential role in the study of the convergence of any sequence of linear positive operators towards the identity operator. For the operators defined by (1.2), the following identities

$$
P_{n}^{[\beta]} e_{0}=e_{0}, P_{n}^{[\beta]} e_{1}=\frac{1}{1-\beta} e_{1}, P_{n}^{[\beta]} e_{2}=\frac{1}{(1-\beta)^{2}} e_{2}+\frac{1}{n(1-\beta)^{3}} e_{1},
$$

take place, see [8, Eqs. (2.13)-(2.14)]. We mention that using the Stirling numbers of the second kind, all $P_{n}^{[\beta]} e_{j}$ moments were explicitly calculated in [1, Proposition 1].

Many classical linear positive operators preserve $e_{0}$ and $e_{1}$, which implies that they have affine functions as fixed points. Such operators are also called Markov type. This property becomes useful in the study of the approximation properties which the operators enjoy. Pursuing this goal, in [7] the authors introduced and investigated the following variant of Jain operators

$$
\left(D_{n}^{[\beta]} f\right)(x)=\sum_{k=0}^{\infty} w_{\beta}\left(k ; u_{n}(x)\right) f\left(\frac{k}{n}\right), f \in C\left(\mathbb{R}_{+}\right), x \geq 0,
$$

where $u_{n}(x)=n(1-\beta) x, x \geq 0$. The following identities

$$
D_{n}^{[\beta]} e_{0}=e_{0}, D_{n}^{[\beta]} e_{1}=e_{1}, D_{n}^{[\beta]} e_{2}=e_{2}+\frac{1}{n(1-\beta)^{2}} e_{1},
$$

hold, see [7, Lemma 2.1].

We remind that for $\beta=0, P_{n}^{[0]}=D_{n}^{[0]}, n \geq 1$, turn into well-known SzászMirakjan operators, see [14], [11].

The aim of this paper is to define an integral Kantorovich-type generalization of $D_{n}^{[\beta]}, n \geq 1$, operators and to establish some approximation properties. These will be achieved in the next two sections.

We specify that we wished the presentation to be self-contained to be accessible to a wide audience.

\section{Integral type construction}

Kantorovich-type constructions are based on replacing the values of the function $f$ on the nodes $k / n, k \geq 0$, with average values of the function obtained by integrals on intervals of the form $I_{n, k}=\left[\frac{k}{n}, \frac{k+1}{n}\right], k \geq 0$. The utility of this type of operators is given by the fact that such classes can approximate functions belonging to larger spaces. 
The first approach in Kantorovich sense of $P_{n}^{[\beta]}, n \geq 1$, operators was achieved by Umar and Razi [15]. They defined and analyzed the operators given by the formula

$$
\left(\widetilde{P}_{n}^{[\beta]} f\right)(x)=n \sum_{k=0}^{\infty} w_{\beta}(k ; n x) \int_{k / n}^{(k+1) / n} f(t) d t
$$

where $f$ is locally integrable function and the right hand side of relation (2.1) is finite.

Our proposal for an integral extension of the operators defined by (1.4) has the following form

$$
\left(\widetilde{D}_{n}^{[\beta]} f\right)(x)=\frac{1}{\lambda_{n}} \sum_{k=0}^{\infty} w_{\beta}\left(k ; u_{n}(x)\right) \int_{k \lambda_{n}}^{(k+1) \lambda_{n}} f(t) d t, x \geq 0,
$$

where

(i) $\left(\lambda_{n}\right)_{n \geq 1}$ is a sequence of strictly decreasing positive numbers such that $\lim _{n \rightarrow \infty} \lambda_{n}=0$,

(ii) $f$ belongs to the space of integrable functions defined on $\mathbb{R}_{+}$such that the series in (2.2) is absolutely convergent.

In the above construction we used a flexible net on $\mathbb{R}_{+}$namely $\left(k \lambda_{n}\right)_{k \geq 0}$. The operators also admit the integral representation

$$
\left(\widetilde{D}_{n}^{[\beta]} f\right)(x)=\int_{0}^{\infty} K_{n}^{*}(x, t) f(t) d t, x \geq 0,
$$

with the kernel

$$
K_{n}^{*}(x, t)=\frac{1}{\lambda_{n}} \sum_{k=0}^{\infty} w_{\beta}\left(k ; u_{n}(x)\right) \chi_{n, k}(t),
$$

where $\chi_{n, k}$ is the characteristic function of the interval $\left[k \lambda_{n},(k+1) \lambda_{n}\right]$ with respect to $\mathbb{R}_{+}, k \geq 0$.

For particular case $\lambda_{n}=\frac{1}{n}$ and $u_{n}(x):=n x$, we reobtain the operators defined at (2.1). Further, if we choose $\beta=0$ in (2.1), the operators turn into Szász-MirakjanKantorovich operators introduced by Butzer [4, Eq. (5)]. Clearly, for each $n \in \mathbb{N}, \widetilde{D}_{n}^{[\beta]}$ is a linear positive operator. In what follows we establish some computational results.

Lemma 2.1. Let $\widetilde{D}_{n}^{[\beta]}, n \in \mathbb{N}$, be the operators defined by (2.2). For each $n \in \mathbb{N}$ the following identities

$$
\begin{gathered}
\widetilde{D}_{n}^{[\beta]} e_{0}=e_{0} \\
\widetilde{D}_{n}^{[\beta]} e_{1}=n \lambda_{n} e_{1}+\frac{1}{2} \lambda_{n} \\
\widetilde{D}_{n}^{[\beta]} e_{2}=\left(n \lambda_{n}\right)^{2} e_{2}+n \lambda_{n}^{2}\left(1+\frac{1}{(1-\beta)^{2}}\right) e_{1}+\frac{1}{3} \lambda_{n}^{2}
\end{gathered}
$$

take place. 
Proof. Taking in view the definition of $D_{n}^{[\beta]}$ operators and identity (1.1), we immediately deduce $\widetilde{D}_{n}^{[\beta]} e_{0}=D_{n}^{[\beta]} e_{0}$, as well as the following identities

$$
\begin{aligned}
& \left(\widetilde{D}_{n}^{[\beta]} e_{1}\right)(x)=n \lambda_{n}\left(D_{n}^{[\beta]} e_{1}\right)(x)+\frac{1}{2} \lambda_{n}\left(D_{n}^{[\beta]} e_{0}\right)(x), \\
& \left(\widetilde{D}_{n}^{[\beta]} e_{2}\right)(x)=\left(n \lambda_{n}\right)^{2}\left(D_{n}^{[\beta]} e_{2}\right)(x)+n \lambda_{n}^{2}\left(D_{n}^{[\beta]} e_{1}\right)(x)+\frac{1}{3} \lambda_{n}^{2}\left(D_{n}^{[\beta]} e_{0}\right)(x) .
\end{aligned}
$$

Using (1.5), the proof is ended.

We indicate the first two central moments of the operators. Set $\varphi_{x}(t)=t-x$, $(t, x) \in \mathbb{R}_{+} \times \mathbb{R}_{+}$.

$$
\begin{aligned}
& \left(\widetilde{D}_{n}^{[\beta]} \varphi_{x}\right)(x)=\left(n \lambda_{n}-1\right) x+\frac{1}{2} \lambda_{n}, \\
& \left(\widetilde{D}_{n}^{[\beta]} \varphi_{x}^{2}\right)(x)=\left(n \lambda_{n}-1\right)^{2} x^{2}+n \lambda_{n}^{2}\left(1+\frac{1}{(1-\beta)^{2}}-\frac{1}{n \lambda_{n}}\right) x+\frac{1}{3} \lambda_{n}^{2} .
\end{aligned}
$$

We recall the Bohman-Korovkin criterion. Briefly speaking, this theorem says: if a sequence of linear and positive operators approximates uniformly the test functions $e_{k}, k=\overline{0,2}$, then it approximates all continuous functions defined on a compact interval.

Remark 2.2. Based on Bohman-Korovkin theorem, studying relations (1.3) it can be observed that the sequence $\left(P_{n}^{[\beta]}\right)_{n \geq 1}$ does not tend to the identity operator. To turn it into an approximation process we will proceed as follows. For each $n \in \mathbb{N}$, the constant $\beta$ will be replaced by a number $\beta_{n} \in[0,1)$. If $\lim _{n \rightarrow \infty} \beta_{n}=0$, then

$$
\lim _{n \rightarrow \infty}\left(P_{n}^{\left[\beta_{n}\right]} e_{j}\right)(x)=e_{j}(x), j \in\{0,1,2\},
$$

uniformly on any compact interval $K \subset \mathbb{R}_{+}$. Consequently,

$$
\lim _{n \rightarrow \infty}\left(P_{n}^{\left[\beta_{n}\right]} f\right)(x)=f(x) \text {, uniformly in } x \in K \text {. }
$$

What is important to point out is that for the Kantorovich variant defined at (2.2) we no longer have to make this change on the $\beta$ parameter, as will be seen in the next paragraph.

\section{Approximation properties of $\widetilde{D}_{n}^{[\beta]}$ operators}

We establish sufficient conditions for the sequence $\left(\widetilde{D}_{n}^{[\beta]}\right)_{n \geq 1}$ to become an approximation process in a certain specified space.

Throughout the paragraph we use standard notations. Set $B(X)$ the Banach space of all real-valued bounded functions defined on $X$, endowed with the norm of the uniform convergence (briefly, sup-norm) defined by $\|f\|=\sup _{x \in X}|f(x)|$ for every $f \in B(X)$. Also, set $C_{B}(X)=C(X) \cap B(X)$, endowed with the sup-norm.

We mention that the operators $\widetilde{D}_{n}^{[\beta]}, n \geq 1$, are non expansive in the space $B\left(\mathbb{R}_{+}\right)$, this means

$$
\left\|\widetilde{D}_{n}^{[\beta]} f\right\| \leq\|f\|,
$$


for any local integrable function $f$ belonging to $B\left(\mathbb{R}_{+}\right)$. As a consequence, for each $n \in \mathbb{N}, \widetilde{D}_{n}^{[\beta]}$ maps continuously $C_{B}\left(\mathbb{R}_{+}\right)$into itself.

Theorem 3.1. Let the operators $\widetilde{D}_{n}^{[\beta]}, n \in \mathbb{N}$, be defined by (2.2) such that the following condition

$$
\lim _{n \rightarrow \infty} n \lambda_{n}=1
$$

is fulfilled. For any compact interval $K \subset \mathbb{R}_{+}$, the following relation

$$
\lim _{n \rightarrow \infty} \widetilde{D}_{n}^{[\beta]} f=f \text { uniformly on } K
$$

occurs, provided $f$ is continuous and bounded on $\mathbb{R}_{+}$.

Proof. A direct way to prove this result is to use a general result established by Altomare [3, Theorem 4.1] which says

Let $X$ be a locally compact subset of $\mathbb{R}^{d}, d \geq 1$. Consider a lattice subspace $E$ of $F(X)$ containing the set $\mathcal{T}=\left\{\mathbf{1}, p r_{1}, \ldots, p r_{d}, \sum_{i=1}^{d} p r_{j}^{2}\right\}$ and let $\left(L_{n}\right)_{n \geq 1}$ be a sequence of positive linear operators from $E$ into $F(X)$ such that for every $g \in \mathcal{T}$,

$$
\lim _{n \rightarrow \infty} L_{n}(g)=g \text { uniformly on compact subsets of } X \text {. }
$$

Then, for every $f \in E \cap C_{B}(X)$,

$$
\lim _{n \rightarrow \infty} L_{n}(f)=f \text { uniformly on compact subsets of } X \text {. }
$$

In the above $F(X)$ stands for the linear space of all real-valued functions defined on $X$ and the function $p r_{j}: \mathbb{R}^{d} \rightarrow \mathbb{R}$ indicates the $j$-th coordinate function,

$$
\operatorname{pr}_{j}(x)=x_{j}, x=\left(x_{j}\right)_{1 \leq j \leq d} \in \mathbb{R}^{d} .
$$

Applying the above result for $d=1, X=\mathbb{R}_{+}, E=C\left(\mathbb{R}_{+}\right)$, the set $\mathcal{T}$ will consist of the test functions $e_{j}, j \in\{0,1,2\}$. The formulas (2.3)-(2.5) correlated with hypothesis (3.2) complete the proof.

In order to obtain the error of approximation we use the modulus of continuity defined as follows

$$
\begin{aligned}
\omega_{f}(\delta) \equiv \omega(f ; \delta) & =\sup \left\{\left|f\left(x^{\prime}\right)-f\left(x^{\prime \prime}\right)\right|: x^{\prime}, x^{\prime \prime} \in \mathbb{R}_{+},\left|x^{\prime}-x^{\prime \prime}\right| \leq \delta\right\} \\
& =\sup _{0 \leq h \leq \delta} \sup _{x \in \mathbb{R}_{+}}|f(x+h)-f(x)|,
\end{aligned}
$$

where $\delta \geq 0$ and $f \in B\left(\mathbb{R}_{+}\right)$.

Theorem 3.2. Let the operators $\widetilde{D}_{n}^{[\beta]}, n \in \mathbb{N}$, be defined by (2.2). For any local integrable function defined on $\mathbb{R}_{+}$belonging to $B\left(\mathbb{R}_{+}\right)$, we get

$$
\left|\left(\widetilde{D}_{n}^{[\beta]} f\right)(x)-f(x)\right| \leq\left(1+\sqrt{\tau(x)+\lambda_{n}^{2} \alpha_{n}^{-1}}\right) \omega\left(f ; \sqrt{\alpha_{n}}\right), x \geq 0,
$$

where

$$
\tau(x)=\max _{x \geq 0}\left\{x, x^{2}\right\} \text { and } \alpha_{n}=\left(n \lambda_{n}-1\right)^{2}+\left(1+\frac{1}{(1-\beta)^{2}}\right) n \lambda_{n}^{2}, n \geq 1 .
$$


Proof. To achieve the statement, we appeal to an old result established by Shisha and Mond [13]: if $T$ is a linear and positive operator, then one has

$$
\begin{aligned}
|(T f)(x)-f(x)| & \leq|f(x)|\left|\left(T e_{0}\right)(x)-1\right| \\
& +\left(\left(T e_{0}\right)(x)+\frac{1}{\delta} \sqrt{\left(T e_{0}\right)(x)\left(T \varphi_{x}^{2}\right)(x)}\right) \omega(f ; \delta), \delta>0,
\end{aligned}
$$

for every bounded function $f$. Using this inequality for $\widetilde{D}_{n}^{[\beta]}$ operators, we take into account relation (2.3). Based on (2.6), we can write

$$
\left(\widetilde{D}_{n}^{[\beta]} \varphi_{x}^{2}\right)(x) \leq \alpha_{n} \tau(x)+\lambda_{n}^{2}, n \geq 1 .
$$

Choosing $\delta:=\sqrt{\alpha_{n}}$ we arrive at $(3.3)$ and the proof is over.

Remark 3.3. Let us suppose that $f$ is uniformly continuous on $\mathbb{R}_{+}$. In this case it is known that $\lim _{\delta \rightarrow 0^{+}} \omega(f ; \delta)=0$, see, e.g., the monograph [5, page 40]. If we request that the condition (3.2) to be fulfilled, then relation (3.3) leads to the fact that $\left(\left(\widetilde{D}_{n}^{[\beta]} f\right)(x)\right)_{n \geq 0}$ is pointwise convergent to $f(x)$ for any $x \in \mathbb{R}_{+}$. Also, $(3.2)$ guarantees that the upper-bound for the error of approximation has the magnitude $\mathcal{O}(1 / \sqrt{n})$.

As a special case we indicate the rate of convergence of our operators by means of the elements of $\gamma$-Hölder continuous class

$$
\operatorname{Lip}_{M}(\gamma)=\left\{f: \mathbb{R}_{+} \rightarrow \mathbb{R}|| f(t)-f(x)|\leq M| t-\left.x\right|^{\gamma},(t, x) \in \mathbb{R}_{+} \times \mathbb{R}_{+}\right\},
$$

where $0<\gamma \leq 1$ and $M$ is a nonnegative constant independent of $f$.

Theorem 3.4. Let the operators $\widetilde{D}_{n}^{[\beta]}, n \in \mathbb{N}$, be defined by (2.2). For any function $f \in \operatorname{Lip}_{M}(\gamma)$ the following inequality

$$
\left|\left(\widetilde{D}_{n}^{[\beta]} f\right)(x)-f(x)\right| \leq M\left(\alpha_{n} \tau(x)+\lambda_{n}^{2}\right)^{\gamma / 2}, x \geq 0,
$$

holds, where $\tau$ and $\alpha_{n}$ are defined at (3.4).

Proof. Considering relations (2.3) and (3.6) we can write

$$
\left|\left(\widetilde{D}_{n}^{[\beta]} f\right)(x)-f(x)\right| \leq \widetilde{D}_{n}^{[\beta]}(|f-f(x)| ; x) \leq M \widetilde{D}_{n}^{[\beta]}\left(\left|\varphi_{x}\right|^{\gamma} ; x\right) .
$$

At this point we apply Hölder inequalities with conjugate numbers

$$
p:=2 / \gamma, q:=2 /(2-\gamma)
$$

inferring

$$
\int_{k \lambda_{n}}^{(k+1) \lambda_{n}}\left|\varphi_{x}(t)\right|^{\gamma} d t \leq \lambda_{n}^{\frac{2-\gamma}{2}}\left(\int_{k \lambda_{n}}^{(k+1) \lambda_{n}} \varphi_{x}^{2}(t) d t\right)^{\gamma / 2}
$$

Returning at (3.8) we get

$$
\begin{aligned}
\left|\left(\widetilde{D}_{n}^{[\beta]} f\right)(x)-f(x)\right| & \leq M \sum_{k=0}^{\infty} w_{\beta}\left(k ; u_{n}(x)\right)\left(\frac{1}{\lambda_{n}} \int_{k \lambda_{n}}^{(k+1) \lambda_{n}} \varphi_{x}^{2}(t) d t\right)^{\gamma / 2} \\
& \leq M\left(\widetilde{D}_{n}^{[\beta]} \varphi_{x}^{2}\right)^{\gamma / 2}(x) .
\end{aligned}
$$

Using the inequality (3.5) we reach (3.7) and the proof is complete. 
We can also consider functions satisfying another Lipschitz type condition defined by Szász [14, Eq. (8)]. Set

$$
\operatorname{Lip}_{M}^{*}(\gamma)=\left\{f: \mathbb{R}_{+} \rightarrow \mathbb{R}|| f(t)-f(x) \mid \leq M \frac{|t-x|^{\gamma}}{(t+x)^{\gamma / 2}}, t>0, x>0\right\},
$$

where $0<\gamma \leq 1$ and $M$ is a nonnegative constant independent of $f$.

Since $(t+x)^{-\gamma / 2}<x^{-\gamma / 2}$, for $t>0$ and $x>0$, following a demonstration path similar to that indicated in Theorem 3.4, we can state

Remark 3.5. For any function $f \in \operatorname{Lip}_{M}^{*}(\gamma)$ it takes place

$$
\left|\left(\widetilde{D}_{n}^{[\beta]} f\right)(x)-f(x)\right| \leq \frac{M}{x^{\gamma / 2}}\left(\alpha_{n} \tau(x)+\lambda_{n}^{2}\right)^{\gamma / 2}, x>0,
$$

where $\tau$ and $\alpha_{n}$ are defined at (3.4).

We focus on establishing the degree of approximation in terms of the second modulus of smoothness $\omega_{2}(f ; \cdot)$ of a function $f \in C_{B}\left(\mathbb{R}_{+}\right)$. It is given as follows

$$
\omega_{2}(f ; \delta)=\sup _{0 \leq h \leq \delta} \sup _{x \geq 0}|f(x+2 h)-2 f(x+h)+f(x)|, \delta \geq 0 .
$$

Also, a subtle measurement of the error of approximation is provided by K-functional introduced by Peetre [12]. If $X_{0}, X_{1}$ are two Banach spaces with $X_{1}$ continuously embedded in $X_{0}, X_{1} \hookrightarrow X_{0}$, the K-functional is defined for each $f \in X_{0}$ by the formula

$$
K\left(f, \delta ; X_{0}, X_{1}\right) \equiv K(f ; \delta)=\inf _{g \in X_{1}}\left(\|f-g\|_{X_{0}}+\delta\|g\|_{X_{1}}\right), \delta>0 .
$$

This quantity describes properties of approximation of $f \in X_{0}$. More detailed, the inequality $K(f ; \delta)<\varepsilon$ for $\delta>0$ implies that $f$ can be approximated with the error $\|f-g\|_{X_{0}}<\varepsilon$ in $X_{0}$ by an element $g \in X_{1}$ whose norm is not too large, namely $\|g\|_{X_{1}}<\varepsilon \delta^{-1}$. For our purpose, we choose

$$
X_{0}=C_{B}\left(\mathbb{R}_{+}\right), X_{1}=C_{B}^{2}\left(\mathbb{R}_{+}\right)=\left\{g \in C_{B}\left(\mathbb{R}_{+}\right): g^{\prime}, g^{\prime \prime} \in C_{B}\left(\mathbb{R}_{+}\right)\right\},
$$

both spaces being endowed with the sup-norm $\|\cdot\|$. Thus, we will use

$$
K(f ; \delta)=\inf _{g \in C_{B}^{2}\left(\mathbb{R}_{+}\right)}\left(\|f-g\|+\delta\left\|g^{\prime \prime}\right\|\right) .
$$

Based for example on [10, Proposition 6.1], between $\omega_{2}$ and K-functional the following relations hold: the positive constants $c_{1}$ and $c_{2}$ exist such that

$$
c_{1} \omega_{2}(f ; \delta) \leq K\left(f ; \delta^{2}\right) \leq c_{2} \omega_{2}(f ; \delta), \delta>0 .
$$

Theorem 3.6. Let the operators $\widetilde{D}_{n}^{[\beta]}, n \in \mathbb{N}$, be defined by (2.2).

For every $f \in C_{B}\left(\mathbb{R}_{+}\right)$the following inequality

$$
\left|\left(\widetilde{D}_{n}^{[\beta]} f\right)(x)-f(x)\right| \leq M \omega_{2}\left(f ; \delta_{n}(x)\right)+\omega\left(f ;\left(n \lambda_{n}-1\right) x+\frac{1}{2} \lambda_{n}\right), x \geq 0,
$$

holds, where $M$ is a constant independent of $f$ and

$$
\delta_{n}(x)=\frac{1}{2}\left(\alpha_{n} \tau(x)+\lambda_{n}^{2}+\left(\left(n \lambda_{n}-1\right) x+\frac{1}{2} \lambda_{n}\right)^{2}\right)^{1 / 2} .
$$


The quantities $\alpha_{n}$ and $\tau$ are defined by (3.4).

Proof. Our approach follows a route similar to the proof that appears in $[6$, Theorem 3.2] aiming at a Kantorovich modification for Szász-Mirakjan operators based on Jain and Pethe operators [9].

At first we define the operators $E_{n}^{[\beta]}: C_{B}\left(\mathbb{R}_{+}\right) \rightarrow C\left(\mathbb{R}_{+}\right)$,

$$
\left(E_{n}^{[\beta]} f\right)(x)=\left(\widetilde{D}_{n}^{[\beta]} f\right)(x)-f\left(n \lambda_{n} x+\frac{1}{2} \lambda_{n}\right)+f(x) .
$$

Using relations (2.3) and (2.4), obviously $E_{n}^{[\beta]} e_{k}=e_{k}$ for $k \in\{0,1\}$. Therefore, the first central moment $E_{n}^{[\beta]} \varphi_{x}$ is null. Since $\widetilde{D}_{n}^{[\beta]}$ verifies (3.1), we have

$$
\left|\left(E_{n}^{[\beta]} h\right)(x)\right| \leq 3\|f\|, x \geq 0,
$$

for any $h \in C_{B}\left(\mathbb{R}_{+}\right)$. Let $g \in C_{B}^{2}\left(\mathbb{R}_{+}\right)$be arbitrarily chosen. By Taylor's expansion with integral form of the remainder, we get

$$
g(t)=g(x)+(t-x) g^{\prime}(x)+\int_{x}^{t}(t-u) g^{\prime \prime}(u) d u,
$$

for $t \geq 0$ and $x \geq 0$. Applying $E_{n}^{[\beta]}$ on both sides, we can write successively

$$
\begin{aligned}
\left(E_{n}^{[\beta]} g\right)(x)-g(x) & =g^{\prime}(x)\left(E_{n}^{[\beta]} \varphi_{x}\right)(x)+E_{n}^{[\beta]}\left(\int_{x e_{0}}^{e_{1}}\left(e_{1}-u e_{0}\right) g^{\prime \prime}(u) d u ; x\right) \\
& =\widetilde{D}_{n}^{[\beta]}\left(\int_{x e_{0}}^{e_{1}}\left(e_{1}-u e_{0}\right) g^{\prime \prime}(u) d u ; x\right) \\
& -\int_{x}^{n \lambda_{n} x+\frac{1}{2} \lambda_{n}}\left(n \lambda_{n} x+\frac{1}{2} \lambda_{n}-u\right) g^{\prime \prime}(u) d u .
\end{aligned}
$$

In the above we used (3.11). Considering the increase

$$
\left|\int_{x}^{t} \varphi_{x}(u) g^{\prime \prime}(u) d u\right| \leq\left\|g^{\prime \prime}\right\|\left|\int_{x}^{t}\right| u-x|d u| \leq\left\|g^{\prime \prime}\right\| \varphi_{x}^{2}(t)
$$

it allows us to write

$$
\left|\left(E_{n}^{[\beta]} g\right)(x)-g(x)\right| \leq\left\|g^{\prime \prime}\right\|\left(\left(\widetilde{D}_{n}^{[\beta]} \varphi_{x}^{2}\right)(x)+\left(\left(n \lambda_{n}-1\right) x+\frac{1}{2} \lambda_{n}\right)^{2}\right) .
$$

Returning at $(3.11)$, with the help of $(3.12)$, definition of modulus of continuity $\omega(f ; \cdot)$ and (3.5), we get

$$
\begin{aligned}
& \left|\left(\widetilde{D}_{n}^{[\beta]} f\right)(x)-f(x)\right| \\
\leq & \left|E_{n}^{[\beta]}(f-g ; x)\right|+\left|\left(E_{n}^{[\beta]} g\right)(x)-g(x)\right|+|g(x)-f(x)|+\left|f\left(n \lambda_{n} x+\frac{1}{2} \lambda_{n}\right)-f(x)\right| \\
\leq & 4\|f-g\|+\left(\left(\widetilde{D}_{n}^{[\beta]} \varphi_{x}^{2}\right)(x)+\left(\left(n \lambda_{n}-1\right) x+\frac{1}{2} \lambda_{n}\right)^{2}\right)\left\|g^{\prime \prime}\right\|+\omega\left(f ;\left(n \lambda_{n}-1\right) x+\frac{1}{2} \lambda_{n}\right) \\
\leq & 4\|f-g\|+\left(\alpha_{n} \tau(x)+\lambda_{n}^{2}+\left(\left(n \lambda_{n}-1\right) x+\frac{1}{2} \lambda_{n}\right)^{2}\right)\left\|g^{\prime \prime}\right\|+\omega\left(f ;\left(n \lambda_{n}-1\right) x+\frac{1}{2} \lambda_{n}\right) .
\end{aligned}
$$


Taking infimum with respect to all $g \in C_{B}^{2}\left(\mathbb{R}_{+}\right)$and using (3.9) we arrive at $(3.10)$ which concludes the proof.

Remark 3.7. Based on the fact that $\lim _{n \rightarrow \infty} \lambda_{n}=0$ and relation (3.2) takes place, we deduce $\lim _{n \rightarrow \infty} \delta_{n}(x)=0$ for any $x \geq 0$.

Conclusion. In this article we propose an integral version in Kantorovich sense of a family of discrete operators recently obtained from genuine Jain operators. The proposed construction involves sub-intervals of the form $\left[k \lambda_{n},(k+1) \lambda_{n}\right], k \geq 0$, these being used in several previous studies. A notable aspect in the fact that the proposed integral variant is an approximation process for any fixed parameter $\beta$ belonging to the interval $[0,1)$. In order to have the same quality, in the discrete operators $\beta$ has to be replaced with a sequence of parameters $\left(\beta_{n}\right)_{n \geq 1}$ such that $\lim _{n \rightarrow \infty} \beta_{n}=0$. For the newly created sequence of linear positive operators, the highlighted approximation properties involve locally integrable functions in different functions spaces. Upper bounds of approximation error have been established using the first and second order modulus of smoothness.

\section{References}

[1] Abel, U., Agratini, O., Asymptotic behaviour of Jain operators, Numer. Algor., 71(2016), 553-565.

[2] Agratini, O., A stop over Jain operators and their generalizations, Analele Universitătii de Vest, Timişoara, Seria Matematică-Informatică, 56(2018), f. 2, 28-42.

[3] Altomare, F., Korovkin-type theorems and approximation by positive linear operators, Surveys in Approximation Theory, 5(2010), 92-164.

[4] Butzer, P.L., On the extensions of Bernstein polynomials to the infinite interval, Proc. Amer. Math. Soc., 5(1954), 547-553.

[5] DeVore, R.A., Lorentz, G.G., Constructive Approximation, Grundlehren der mathematischen Wissenschaften, Vol. 303, Springer-Verlag, 1993.

[6] Dhamija, M., Pratap, R., Deo, N., Approximation by Kantorovich form of modified Szász-Mirakyan operators, Appl. Math. Comput., 317(2018), 109-120.

[7] Doğru, O., Mohapatra, R.N., Örkcü, M., Approximation properties of generalized Jain operators, Filomat 30:9(2016), 2359-2366.

[8] Jain, G.C., Approximation of functions by a new class of linear operators, J. Australian Math. Soc., 13(1972), no. 3, 271-276.

[9] Jain, G.C., Pethe, S., On the generalizations of Bernstein and Szász-Mirakjan operators, Nanta Math., 10(1977), 185-193.

[10] Johnen, H., Inequalities connected with the moduli of smoothness, Mat. Vesnik, $\mathbf{9}(\mathbf{2 4})(1972)$, 289-305.

[11] Mirakjan, G.M., Approximation of functions with the aid of polynomials, (in Russian), Dokl. Akad. Nauk SSSR, 31(1941), 201-205.

[12] Peetre, J., A theory of interpolation of normed spaces, Notas de Matemática, Rio de Janeiro, 39(1968), 1-86. 
[13] Shisha, O., Mond, B., The degree of convergence of linear positive operators, Proc. Nat. Acad. Sci. USA, 60(1968), 1196-1200.

[14] Szász, O., Generalization of S. Bernstein's polynomials to the infinite interval, J. Res. Nat. Bur. Standards, 45(1950), 239-245.

[15] Umar, S., Razi, Q., Approximation of functions by generalized Szász operators, Commun. Fac. Sci. de l'Université d'Ankara, Séries $A_{1}$ : Mathématique, 34(1985), 45-52.

Octavian Agratini

Babeş-Bolyai University, Faculty of Mathematics and Computer Science, Str. Kogălniceanu, 1, 400084 Cluj-Napoca, Romania

and

Tiberiu Popoviciu Institute of Numerical Analysis, Romanian Academy, Str. Fântânele, 57, 400320 Cluj-Napoca, Romania e-mail: agratini@math.ubbcluj.ro

Ogun Doğru

Department of Mathematics, Faculty of Science, Gazi University, Ankara, Turkey

e-mail: ogun.dogru@gazi.edu.tr 